\title{
Escore de Risco Clínico Simples para Prever a Mortalidade Pós-Alta Hospitalar em Pacientes Chineses Hospitalizados por Insuficiência Cardíaca
}

A Simple Clinical Risk Score to Predict Post-Discharge Mortality in Chinese Patients Hospitalized with Heart Failure

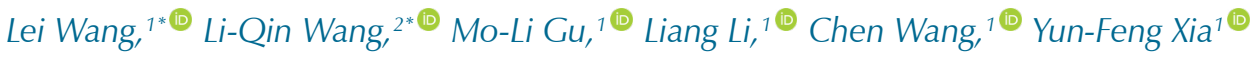 \\ Departamento de Medicina Geriátrica, the Fourth Medical Center, Chinese PLA General Hospital, ${ }^{1}$ Beijng - China \\ Departamento de Enfermagem, the Eighth Medical Center, Chinese PLA General Hospital, ${ }^{2}$ Beijng - China \\ *Os autores contribuíram igualmente para esse artigo
}

\section{Resumo}

Fundamento: Doenças cardiovasculares são a principal causa de morte na China. Entretanto, os esforços atuais para se identificar os fatores de risco de morte em pacientes hospitalizados com insuficiência cardíaca (IC) estão direcionados principalmente para a mortalidade durante a internação e a mortalidade após 30 dias nos Estados Unidos. Dessa forma, é necessário um modelo semelhante ao modelo utilizado para prever o risco considerado para procedimentos cirúrgicos cardiovasculares em pacientes para avaliar o risco de pacientes internados com diagnóstico de IC.

Objetivo: Identificar variáveis que podem prever a mortalidade por IC um ano após a alta hospitalar, e desenvolver um escore de risco para avaliar o risco de morte no período de um ano.

Métodos: No presente estudo, 1.742 pacientes chineses com IC foram divididos aleatoriamente em dois grupos: um grupo de amostra de derivação e um grupo de amostra de teste. O método de simulação Monte Carlo via Cadeias de Markov foi usado para identificar variáveis que podem prever a mortalidade um ano após a alta hospitalar. Variáveis com uma frequência $>1 \%$ na análise bivariada, e que foram consideradas clinicamente significativas, foram qualificadas para análises de modelagens posteriores. A probabilidade posterior de que uma variável estava estatística e significativamente associada ao resultado foi calculada como o número total de vezes em que o IC de $95 \%$ da variável não coincidiu com 1 (ou seja, o ponto de referência), dividido pelo número total de iterações. Uma variável com uma probabilidade de 0,9 ou mais alta foi considerado um fator de risco robusto para prever o resultado, e foi incluída na lista final de variáveis. O nível de significância estatística adotado foi $5 \%$.

Resultados: Cinco variáveis que pudessem prever de maneira robusta a mortalidade um ano após a alta hospitalar foram identificadas: idade, sexo feminino, escore da New York Heart Association (Associação de Cardiologia de Nova lorque) >3, diâmetro do átrio esquerdo, e índice de massa corporal. Os modelos de derivação e de teste tiveram uma área de curva característica de operação do receptor de 0,79. Essas variáveis selecionadas foram utilizadas para avaliar o escore de risco de mortalidade por IC após um ano, e este foi dividido em três grupos (baixo, moderado e alto). O grupo de alto risco corresponde a aproximadamente $86 \%$ das mortes, e o grupo de risco moderado corresponde a $12 \%$ das mortes.

Conclusão: Um escore de risco de 5 variáveis simples pode ser utilizado para avaliar a mortalidade um ano após a alta hospitalar de pacientes internados com IC.

Palavras-chave: Insuficiência Cardíaca, Pontuação de Propensão, Mortalidade, Alta Hospitalar, Epidemiologia

\begin{abstract}
Background: Cardiovascular diseases are the leading causes of death in China. However, present efforts to identify the risk factors for death in patients hospitalized with heart failure (HF) are primarily focused on in-hospital mortality and 30-day mortality in the United States. Thus, a model similar to the model used for predicting the risk in patients considered for cardiovascular surgical procedures is needed to evaluate the risk of the patients admitted with a diagnosis of HF.
\end{abstract}

Objective: To identify variables that can predict post-discharge one-year HF mortality and develop a risk score to assess the risk of dying within one year.

Correspondência: Xia Yunfeng •

Fourth Medical Center of PLA General Hospital - No.51 Fucheng Road, Haidian District Beijing 100048 - China

E-mail: yunfengxia111@163.com

Artigo recebido em 07/05/2020, revisado em 10/09/2020, aceito em 04/11/2020

DOI: https://doi.org/10.36660/abc.20200435 
Methods: In the present study, 1,742 Chinese patients with HF were randomly divided into two groups: a derivation sample group and a test sample group. A Markov Chain Monte Carlo simulation method was used to identify variables that can predict the one-year post-discharge mortality. Variables with a frequency of $>1 \%$ in the bivariate analysis and that were considered clinically meaningful were eligible for further modeling analyses. The posterior probability that a variable was statistically and significantly associated with the outcome was calculated as the total number of times that the variable's $95 \% \mathrm{Cl}$ did not overlap with 1 (i.e., the reference point) divided by the total number of iterations. A variable with a probability of 0.9 or higher was considered a robust risk factor for predicting the outcome, and this was included in the final variable list. The level of statistical significance adopted was 5\%.

Results: Five variables that could robustly predict the one-year post-discharge mortality were identified: age, female gender, New York Heart Association functional classification score $>3$, left atrial diameter, and body mass index. Both derivation and test models had a receiver operating curve area of 0.79. These selected variables were used to assess the one-year HF mortality risk score, and these were divided into three groups (low, moderate, and high). The high-risk group corresponds to nearly $86 \%$ of the deaths, while the moderate group corresponds to $12 \%$ of the deaths.

Conclusion: A simple 5-variable risk score can be used to assess the one-year post-discharge mortality of hospitalized Chinese patients with HF. Keywords: Heart Failure; Propensity Score; Mortality; Patient Discharge; Epidemiology.

Full texts in English - http://www.arquivosonline.com.br

\section{Introdução}

Doenças cardiovasculares são a principal causa de morte na China, correspondendo a aproximadamente $22,5 \%$ de todas as mortes. ${ }^{1}$ A insuficiência cardíaca (IC) é a décima-segunda principal causa de internação hospitalar na China, e quatro milhões de chineses sofrem dessa doença. ${ }^{1}$ No geral, a IC na China tem um prognóstico especialmente ruim, com até $40 \%$ dos pacientes morrendo por IC no período de um ano. ${ }^{1}$ A carga financeira da IC também é considerável. ${ }^{2}$

Entretanto, os esforços atuais para se identificar os fatores de risco de morte em pacientes hospitalizados por IC, tais como o escore de risco de Framingham, estão voltados principalmente para a mortalidade durante a internação hospitalar ${ }^{3,4}$ e a mortalidade após 30 dias nos Estados Unidos. ${ }^{5,6}$ Como a IC é uma doença crônica, a identificação dos fatores de risco de mortalidade de pacientes com IC no longo prazo poderia beneficiar os pacientes. Um modelo semelhante ao modelo utilizado para prever o risco considerado para procedimentos cirúrgicos cardiovasculares em pacientes pode ser utilizado para avaliar o risco de pacientes internados com diagnóstico de IC. ${ }^{7}$ Considerando-se o aumento da carga de IC na China, é importante encontrar meios para a estratificação de pacientes com base no risco, no momento do diagnóstico inicial e no momento da alta. Além disso, como a população asiática representa aproximadamente 5\% da população dos Estados Unidos e a população chinesa representa $20 \%$ da população mundial, os escores de risco desenvolvidos com base em populações ocidentais geralmente estimam incorretamente o risco para populações asiáticas. ${ }^{8}$ Portanto, é importante desenvolver ferramentas clinicamente relevantes para chineses e outros grupos asiáticos. Uma ferramenta que pudesse especificamente indicar a probabilidade de mortalidade após um ano em pacientes chineses com IC teria grande utilidade clínica, pois teria o potencial de orientar a tomada de decisão clínica e de identificar pacientes que têm mais probabilidade de precisar de um monitoramento pós-alta intenso. Além disso, como a raça, a etnia, e o país de origem têm um grande impacto nos resultados clínicos, é importante desenvolver uma avaliação de risco específica para o grupo de interesse, ou seja, os pacientes chineses com IC.
O presente estudo tem o objetivo de identificar fatores de risco que estão mais fortemente correlacionados à mortalidade após um ano entre pacientes chineses com IC, e de desenvolver um escore de risco simples para avaliar o risco de mortalidade um ano após a alta hospitalar nesses pacientes.

\section{Métodos}

\section{Sujeitos}

O presente estudo foi aprovado pelo Comitê de Ética de nosso hospital, e todos os pacientes assinaram o termo de consentimento informado.

O coorte do estudo foi extraído do Beijing Monitoring Heart Failure Patients and Building Heart Failure Management Network Study (Estudo de monitoramento de pacientes com insuficiência cardíaca e de construção de rede de gestão da insuficiência cardíaca de Pequim), que incluiu todos os pacientes com idade $\geq 20$ anos hospitalizados por IC em dos 14 hospitais designados em Pequim, na China, entre 10 de outubro de 2015 e 9 de outubro de 2017. Esses pacientes foram divididos aleatoriamente em dois grupos usando um método de tabela aleatória: um grupo de amostra de derivação e um grupo de amostra de teste. As informações sobre mortalidade após um ano foram obtidas por entrevistas telefônicas pós-alta hospitalar.

\section{Candidatas a variáveis de risco e variáveis de resultado}

As candidatas a variáveis de risco incluíram as características demográficas (idade, sexo, e índice de massa corporal [IMC]), histórico médico e comorbidades, fatores de estilo de vida, cirurgias cardíacas prévias, achados clínicos e resultados de testes laboratoriais. A idade e o IMC foram medidos como variáveis contínuas, enquanto sexo foi codificado como feminino (sim/não). O histórico médico incluiu histórico de infarto do miocárdio agudo (IMA), histórico de IC, e histórico de doença cardíaca coronária (DCC), diabetes tipo I ou tipo II, e hipertensão. Cirurgias cardíacas prévias incluíram cirurgias de válvula anteriores. Os achados clínicos incluíram o escore 
de classificação funcional da New York Heart Association (NYHA) (classe >3) e fração de ejeção ventricular esquerda $(<40 \%)$. Fatores de estilo de vida incluíram histórico de tabagismo, tabagismo atual e consumo de álcool. Os dados de testes laboratoriais incluíram a frequência cardíaca, a pressão arterial, e o diâmetro do átrio esquerdo (em milímetros), que foi medido como variável contínua.

A variável de resultado foi a mortalidade por IC um ano após a alta hospitalar, definida como morte por qualquer causa que tenha ocorrido após uma internação hospitalar por IC registrada. As informações sobre mortalidade foram obtidas por meio de entrevistas telefônicas com esses pacientes. A última entrevista foi realizada em 19 de fevereiro de 2019. Se um paciente tivesse morrido dentro de um ano após a alta, a data da morte era obtida de familiares. Pacientes foram excluídos da amostra do estudo quando nem eles, nem seus familiares puderam ser contactados.

\section{Análise estatística}

Os dados do grupo de amostra de derivação e o grupo de amostra de teste foram comparados pelo teste qui-quadrado em relação às variáveis categóricas e testes $t$ não pareados em relação às variáveis contínuas. Em seguida, o julgamento clínico e a análise de correlação bivariada de Spearman foram utilizados para identificar candidatas a variáveis que possam estar associadas à mortalidade um ano após a alta hospitalar. Variáveis com uma frequência $>1 \%$ na análise bivariada, e que foram consideradas clinicamente significativas, foram qualificadas para análises de modelagens posteriores. Para as observações em que faltavam dados, foi criada uma variável dummy para atribuir um valor de 0 quando o valor da variável estava presente, e um valor de 1 quando a variável não estava presente. Em seguida, os valores que faltavam foram substituídos pela mediana dos valores presentes daquela variável contínua, e as variáveis contínuas e dummy foram incluídas no modelo. Esse método para modelagem dos dados faltantes considerou que esses dados faltavam aleatoriamente, e permitiu a inclusão de todos os casos disponíveis, embora não tenha sido tão eficiente quanto procedimentos de imputação múltipla.

O método de simulação Monte Carlo via Cadeias de Markov (MCMC) foi usado juntamente com a técnica de regressão logística para identificar um conjunto final de fatores de risco que podem prever a mortalidade por IC um ano após a alta hospitalar. As simulações foram feitas com 10.000 iterações para a amostra de derivação, e um modelo logístico foi ajustado a cada iteração, produzindo um conjunto de variáveis que são "estatisticamente significativas", ou associadas ao resultado. Dessa forma, 10.000 iterações da simulação produziram 10.000 conjuntos de razões de chance (RC) e intervalos de confiança (IC) de 95\%, indicando o nível de significância da associação de cada variável com o resultado. A probabilidade posterior de que uma variável estava estatisticamente e significativamente associada ao resultado foi calculada como o número total de vezes em que o IC de 95\% da variável não coincidiu com 1 (ou seja, o ponto de referência), dividido pelo número total de iterações. Uma variável com uma probabilidade de 0,9 ou mais alto foi considerado um fator de risco robusto para prever o resultado, e foi incluído na lista final de variáveis. Esse método foi utilizado em outros estudos. ${ }^{9}$ A área sob a curva característica de operação do receptor (ROC) foi calculada para cada modelo ajustado por iteração, para se avaliar seu poder discriminatório. ${ }^{10}$

\section{Desenvolvimento do escore de risco}

Com base nos resultados da simulação, foi construído um escore de risco simples, baseado em variáveis selecionadas para avaliar a mortalidade após um ano. Cada variável foi ponderada utilizando-se o coeficiente padronizado (CP) específico da variável obtido de um modelo logístico baseado na amostra de derivação original, com a mortalidade após um ano como resultado, e as variáveis selecionadas como variáveis independentes. $\mathrm{O} C \mathrm{CP}$, que mediu a mudança de coeficiente para uma alteração de desvio padrão (DP) na variável independente, teve o objetivo de representar a importância relativa da variável independente num modelo de regressão. Isso permite fazer a comparação entre variáveis independentes utilizando-se unidades comuns. O escore de risco para cada observação, nas amostras de derivação e de validação, foi calculado como: Escore $=\sum$ Peso $_{i} \cdot$ Variável $_{i}$ em que peso ${ }_{i}=\mathrm{SC}_{\mathrm{i}} / \sum|\mathrm{SC}|, \mathrm{e} \mathrm{i}=1,2,3, \ldots$, número total de variáveis selecionadas finais. Em seguida, cada peso foi redimensionado por 100 para permitir que o escore seja uma prática de fácil utilização, com exceção do peso da idade, que é dimensionado apenas em 10. Cada peso foi posteriormente arredondado para cima ou para baixo, até seu número inteiro mais próximo em intervalos de 5 (por exemplo, 32,5 para 30,0, ou 18,0 para 20,0). A idade foi arredondada para baixo até seu patamar mais próximo, com um ponto decimal. Por último, uma base de 100 foi adicionada ao risco para garantir que não houvesse valores negativos nos escores. Para validar e testar esse escore de risco, dois modelos logísticos foram ajustados. Um modelo utilizou variáveis individuais que foram selecionadas na simulação MCMC como variáveis independentes, e o outro modelo utilizou o escore de risco como variável independente. Esses dois modelos foram ajustados com amostras de derivação e de teste, e os valores de r quadrado e ROC foram calculados de ambos os modelos para avaliar o desempenho do escore de risco. Todos os testes estatísticos foram bilaterais e tiveram um nível de significância de 5\%, e todas as análises foram realizadas utilizando-se a o software SAS versão 9.3 versão de 64 bits (SAS Institute Inc., Cary, Carolina do Norte, EUA). Todas as variáveis contínuas foram distribuídas normalmente como testadas pelos testes de Shapiro-Wilk. As variáveis contínuas com distribuição normal são descritas utilizando-se média e desvio padrão.

\section{Resultados}

\section{Características do paciente}

O coorte final do estudo incluiu um total de 1.742 pacientes com IC. Entre esses pacientes, as amostras de derivação e de teste incluíram 882 e 860 pacientes, respectivamente. A idade média (DP) do coorte foi de 57,0 $(12,5)$ anos, e 9,5\% desses pacientes tinham 40 anos de idade ou menos, enquanto $30,9 \%$ desses pacientes tinham idade igual ou superior a 65 anos. Além disso, 19,9\% desses pacientes eram mulheres. As características dos pacientes nas amostras de derivação e de validação eram comparáveis (Tabela 1). Não houve diferenças significativas entre esses dois grupos em termos de idade, sexo, 


\section{Tabela 1 - Características de linha de base da população do estudo}

\begin{tabular}{|c|c|c|c|c|}
\hline Características do paciente & Geral $(n=1742)$ & $\begin{array}{c}\text { Amostra de } \\
\text { derivação ( } n=882)\end{array}$ & $\begin{array}{l}\text { Amostra de teste } \\
\quad(n=860)\end{array}$ & Valor de $p$ \\
\hline $\begin{array}{l}\text { Dados demográficos } \\
\text { Idade, média (DP) anos }\end{array}$ & $57,1(12,4)$ & $57,0(12,4)$ & $57,1(12,5)$ & 0,9295 \\
\hline Idade faltante, $n=4(\%)$ & 0,2 & 0,2 & 0,2 & 0,9798 \\
\hline Feminino (\%) & 19,9 & 19,8 & 19,9 & 0,9823 \\
\hline IMC, média (DP) & $25,1(3,4)$ & $25,1(3,4)$ & $25,0(3,4)$ & 0,3455 \\
\hline IMC faltante, $\mathrm{n}=0$ (\%) & 0 & 0 & 0 & \\
\hline \multicolumn{5}{|l|}{ Achados clínicos e testes laboratoriais } \\
\hline Diâmetro do átrio esquerdo (mm), média (DP) & $39,9(7,9)$ & $40,0(8,1)$ & $39,9(7,7)$ & 0,823 \\
\hline Tamanho do átrio esquerdo faltante, $n=66(\%)$ & 3,8 & 4,2 & 3,1 & 0,1611 \\
\hline Frequência cardíaca, média (DP) & $73,0(14,0)$ & $73,3(14,8)$ & $72,7(13,2)$ & 0,8762 \\
\hline Frequência cardíaca faltante, n=12 (\%) & 0,7 & 0,5 & 0,9 & 0,2291 \\
\hline Frequência cardíaca >100 (\%) & 4,5 & 5,6 & 3,4 & 0,0276 \\
\hline FEVE $<40(\%)$ & 35,5 & 35,8 & 35,1 & 0,7564 \\
\hline FEVE faltante, $n=0(\%)$ & 0 & 0 & 0 & \\
\hline \multicolumn{5}{|l|}{ Histórico médico } \\
\hline IC (\%) & 18,1 & 20,2 & 15,8 & 0,0177 \\
\hline IMA (\%) & 22,6 & 23,4 & 21,9 & 0,4557 \\
\hline NYHA >3(\%) & 42,7 & 42,6 & 42,8 & 0,9461 \\
\hline DCC (\%) & 71,2 & 70,4 & 72,0 & 0,4699 \\
\hline DM (\%) & 27,8 & 27,2 & 28,4 & 0,5885 \\
\hline TxC (\%) & 63,6 & 62,7 & 64,5 & 0,4258 \\
\hline Cirurgia de válvula (\%) & 6,1 & 6,8 & 5,4 & 0,2044 \\
\hline \multicolumn{5}{|l|}{ Estilo de vida } \\
\hline Tabagismo (\%) & 57,8 & 57,6 & 57,9 & 0,8956 \\
\hline Consumo de álcool (\%) & 35,1 & 34,5 & 35,8 & 0,5561 \\
\hline Mortalidade após um ano (\%) & 6,5 & 7,3 & 5,8 & 0,2236 \\
\hline
\end{tabular}

diâmetro do átrio esquerdo, frequência cardíaca, FEVE <40 (\%), IMA (\%), NYHA > 3 (\%), DCC (\%), DM (\%), HTN (\%), cirurgia de válvula (\%), histórico de tabagismo (\%), consumo de álcool (\%), e mortalidade após um ano (\%). Entretanto, não houve diferenças significativas entre esses dois grupos em termos de frequência cardíaca >100 (\%) e IC (\%). Não houve diferenças significativas em termos de medicamentos e consultas médicas durante o acompanhamento, nem diferenças significativas em resultados de testes laboratoriais entre todos os pacientes.

Variáveis de risco para previsão da mortalidade por IC após um ano

Os índices de mortalidade após um ano observados nas amostras de derivação e validação foram 7,3\% e 5,8\%, respectivamente $(p=0,2236)$. A Figura 1 apresenta a probabilidade de que cada variável seja associada à mortalidade por IC após um ano. Cinco variáveis, incluindo idade, sexo feminino, IMC, diâmetro do átrio esquerdo, e classe NYHA $>3$, tinham uma probabilidade de 0,9 ou mais alta de associação significativa com a mortalidade após um ano. Elas foram identificadas como as variáveis finais (Figuras 1a e 1b). A Tabela 2 ilustra as RC, CP, e IC de 95\% para cada uma das cinco variáveis selecionadas no conjunto de dados de derivação. A área sob a curva ROC desse modelo baseado em 5 variáveis foi 0,789 , com um r quadrado de 0,1761 e uma bondade do ajuste de 0,9013. A capacidade preditiva variou de 0,04, no decil mais baixo, a 0,43 no decil mais alto, indicando que o modelo tem bom poder discriminatório (Figura 1c). Esse modelo também se comportou de maneira semelhante ao conjunto de dados de teste (Figura 1d e Tabela 3).

\section{Escore de risco}

O escore de risco foi construído utilizando-se as estimativas de CP da Tabela 2. A equação de cálculo também está listada 


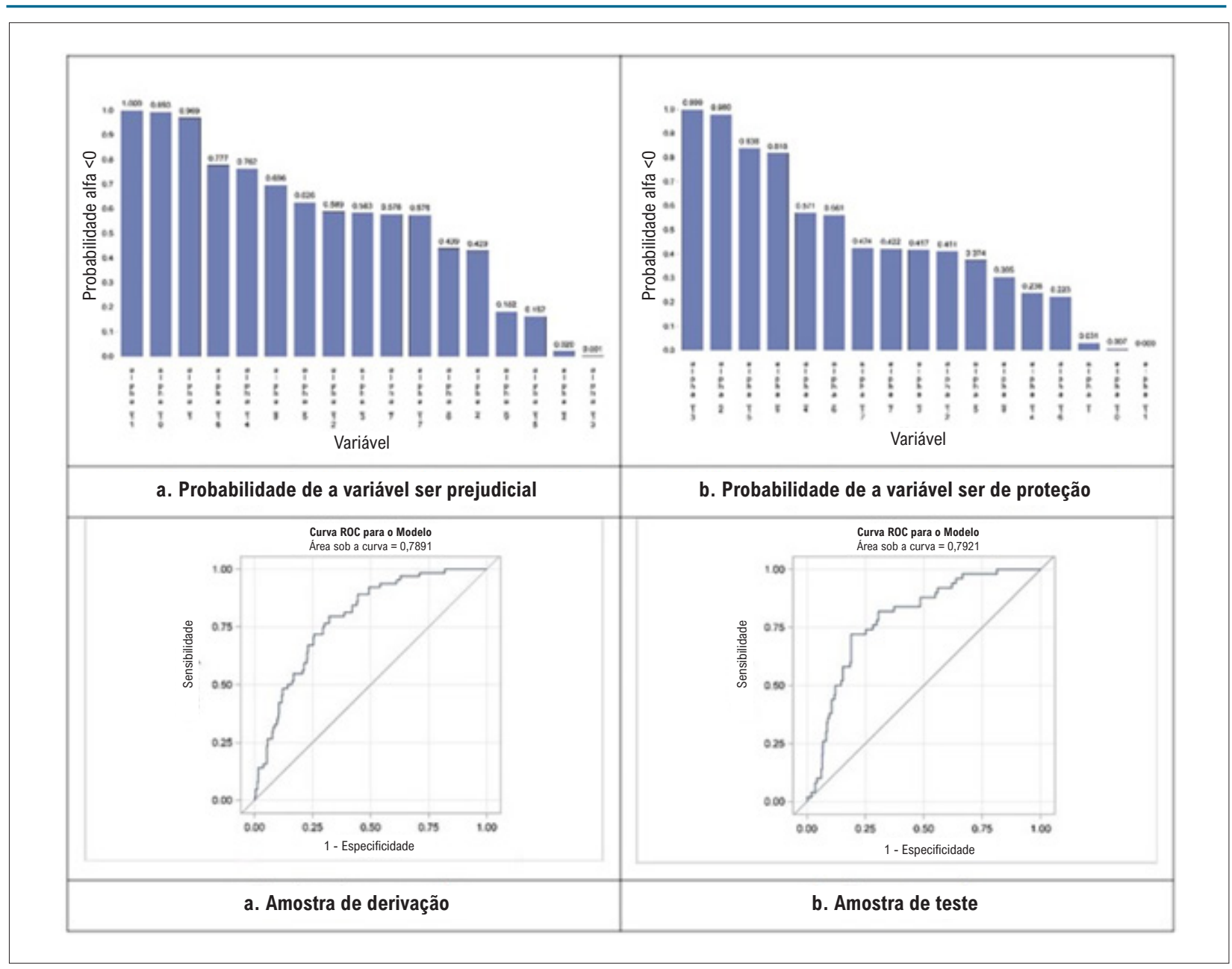

Figura 1 - Seleção variável e desenvolvimento de modelo para escore de risco.

Tabela 2 - Fatores de risco e pesos correspondentes no escore de risco clínico

\begin{tabular}{lcccccr}
\hline Características do paciente & $\begin{array}{c}\text { Probabilidade } \\
\text { posterior* }\end{array}$ & $\begin{array}{c}\text { Razões de chance } \\
\text { (IC 95\%) }\end{array}$ & $\begin{array}{c}\text { Coeficiente } \\
\text { padronizado }\end{array}$ & Peso inicial & Direção & Peso redimensionado \\
\hline Idade, anos & 0,969 & $1,03(1.01-1.05)$ & 0,1864 & 0,155891946 & 1,5 \\
\hline Feminino & 0,02 & $0,44(0.20-0.97)$ & $-0,1796$ & 0,150204901 & $(-)$ & -15 \\
\hline diâmetro do átrio esquerdo $(\mathrm{mm})$ & 0,993 & $1,05(1.02-1.09)$ & 0,2157 & 0,180396421 & 20 \\
\hline IMC & 0,001 & $0,89(0.82-0.96)$ & $-0,2254$ & 0,188508823 & $(-)$ & -20 \\
\hline NYHA $\geq 3$ & 1,000 & $4,2(2.07-8.34)$ & 0,3886 & 0,324997909 & 30 \\
\hline
\end{tabular}

*Probabilidade posterior de que a caracteristica aumente a probabilidade de morte por IC após um ano.

Uso do escore: Score $=100+1.5 a g e-15 f e m a l e ~+20$ leftatrium - 20 BMI + 30 NYHA_3 em que NYHA_3 denota o escore de classificação funcional da New York Heart Association >3. Considerando uma paciente especifica, de 45 anos de idade, do sexo feminino, com IC, IMC de 23, e diâmetro do átrio esquerdo de 30, e classificação NYHA =2, o escore de risco de mortalidade um ano pós-alta hospitalar para essa paciente pode ser calculado utilizando-se a seguinte fórmula: Escore $=100+1,5 \times 45-15 \times 1+20 \times 30-20 \times 23+30 \times 0=322,5$. 0 escore de risco fica entre a faixa de risco baixo e a de risco moderado.

na Tabela 2. A média (DP) do escore de risco baseado em amostra de derivação foi de 492,5 $(177,1)$, com uma faixa de 89,9-1195,63, e a média (DP) do escore baseado em amostra de teste foi de 493,0, com uma faixa de 89,9-1073,9. A diferença em escores médios entre as amostras de derivação e de teste não era estatisticamente significativa $(p=0,7324)$. O escore de risco foi utilizado como variável independente para ajustar a um modelo logístico, o que produziu uma área sob a curva ROC de 0,75 e 0,77 para as amostras de derivação e de teste, respectivamente (Tabela 3). A Figura 2 mostra a distribuição do escore de risco (Figura 2a e 2 b) e a relação exponencial entre o escore e a probabilidade de mortalidade por IC um ano após a alta hospitalar (Figura 2c). 
Tabela 3 - Desempenho do modelo nos diferentes escores de risco

\begin{tabular}{lcccccc}
\hline & & Modelo baseado em 5 fatores de risco & & Modelo baseado em fatores de risco \\
\cline { 2 - 7 } Amostra & ROC & $\begin{array}{c}\text { R-quadrado } \\
\text { redimensionado máximo }\end{array}$ & $\begin{array}{c}\text { Teste de Hosmer } \\
\text { Lemeshow }\end{array}$ & ROC & $\begin{array}{c}\text { R-quadrado } \\
\text { redimensionado } \\
\text { máximo }\end{array}$ & $\begin{array}{c}\text { Teste de Hosmer e } \\
\text { Lemeshow }\end{array}$ \\
\hline Derivação & 0,789 & 0,1761 & 0,9013 & 0,75 & 0,1159 & 0,0243 \\
\hline Teste & 0,792 & 0,1514 & 0,3725 & 0,771 & 0,097 & 0,005 \\
\hline Geral & 0,7858 & 0,1589 & 0,5945 & 0,759 & 0,1069 & 0,003 \\
\hline
\end{tabular}

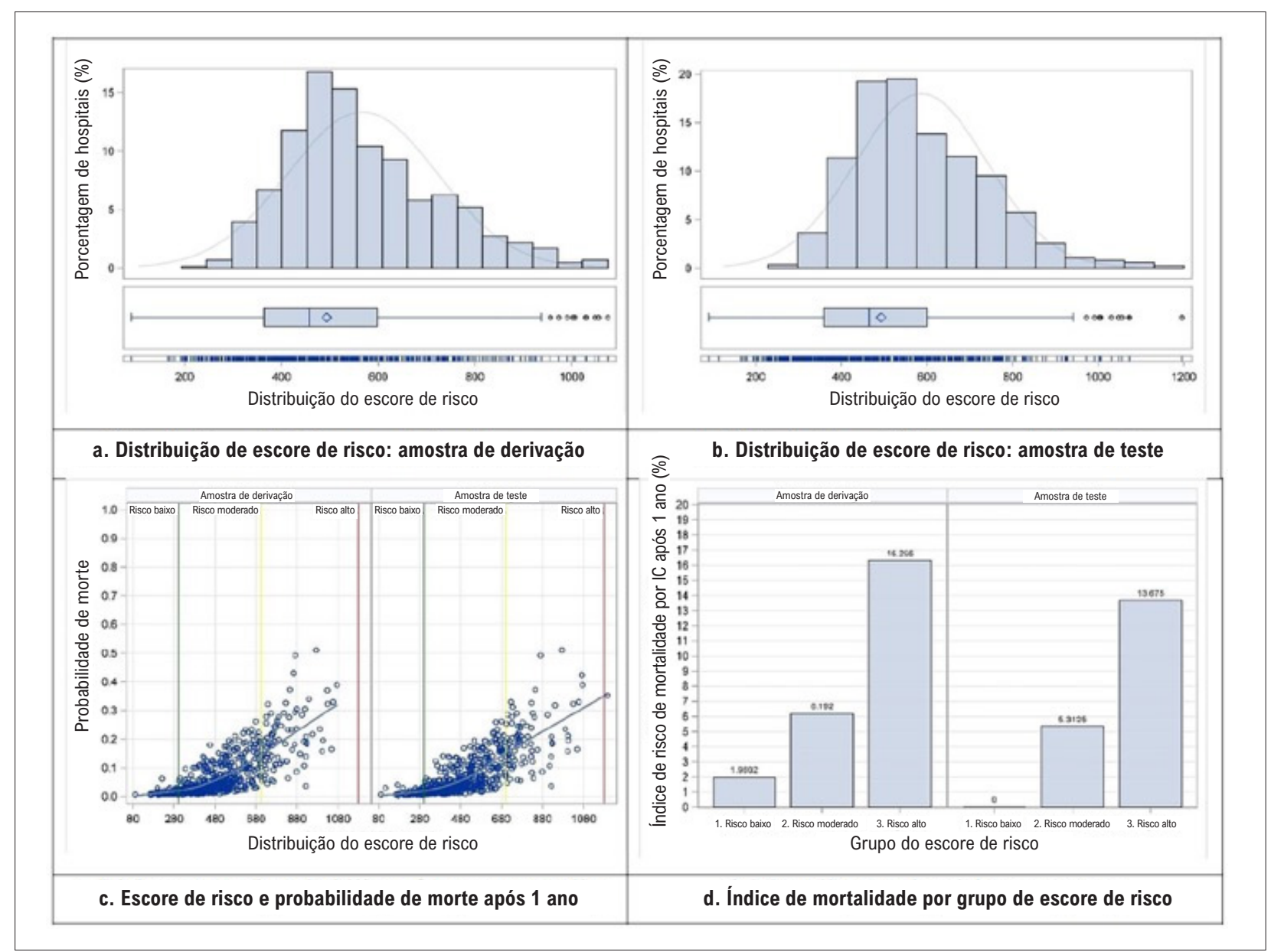

Figura 2 - Distribuição do escore de risco.

O escore de risco tem boa capacidade preditiva. Com a amostra de derivação, as probabilidades médias previstas para mortalidade após um ano foram de 0,15 e 0,07 para aqueles que morreram dentro do período de um ano e aqueles que sobreviveram após o período de um ano, respectivamente. Esse padrão foi semelhante para a amostra de teste, as probabilidades médias previstas para mortalidade após um ano foram de 0,14 e 0,07 para aqueles que morreram e aqueles que sobreviveram, respectivamente. $\mathrm{O}$ escore de risco foi dividido em três faixas baseadas em sua distribuição: (1) risco baixo, se o escore fosse $<300$; (2) risco moderado se o escore fosse $\geq 300$ e $\leq 800$; (3) risco alto se o escore fosse $>800$. As proporções de pacientes em cada um desses três grupos de risco foram as seguintes: $11,7 \%$ para grupos de risco baixo, $73,8 \%$ para grupos de risco moderado, e 14,5\% para grupos de alto risco. A Figura $2 \mathrm{~d}$ mostra as taxas de mortalidade após um ano por risco de grupo e pelas amostras de derivação e de teste. A Figura 3 mostra a distribuição da pontuação de risco por grupos que morreram e sobreviveram.

\section{Discussão}

O desenvolvimento de um modelo de risco de IC específico para a população chinesa é importante, considerando-se que os escores de risco existentes, tais como o amplamente 
utilizado escore de risco de Framingham, são desenvolvidos principalmente nos Estados Unidos, e não conseguem estimar corretamente o risco em populações asiáticas..$^{8-12}$ O presente estudo desenvolveu e validou um escore de risco de 5 variáveis simples para avaliar o risco de mortalidade após um ano em pacientes chineses com IC. Esse escore simples pode ser adicionado à avaliação atual de pacientes antes da alta hospitalar, para proporcionar uma base para que os médicos possam alocar melhor os recursos e identificar pacientes que possam precisar cuidados após a alta. As variáveis finais identificadas no presente estudo foram consistentes com os fatores de risco identificados em coortes ocidentais. Além da idade e da classificação NYHA, que são fatores de risco de mortalidade por IC bem conhecidos, identificou-se que o sexo feminino e o IMC são fatores de proteção dentro do coorte chinês, consistente com os achados baseados na população ocidental. ${ }^{13,14}$ Da mesma forma, identificou-se que o diâmetro do átrio esquerdo estava associado à hospitalização por IC e morte. ${ }^{15}$

O número de pacientes dentro do presente coorte foi semelhante aos escores de risco desenvolvidos para outras populações com IC. ${ }^{16}$ Além disso, em comparação com os estudos anteriores, o presente estudo tem vários pontos fortes. Primeiramente, diferentemente de estudos anteriores que avaliaram o risco de mortalidade em curto prazo em pacientes com IC, o presente estudo focou em resultados em longo prazo. Segundo, estes presentes resultados são específicos para pacientes chineses, já que não são derivados de dados obtidos de outros grupos raciais ou étnicos. Portanto, isto se aplica mais a pacientes chineses, em comparação com escores de risco baseados largamente em coortes ocidentais.
Por exemplo, o tamanho de efeito entre IMC e mortalidade por IC pode variar entre a população ocidental e a chinesa, uma vez que a população chinesa tem um padrão de gordura corporal diferente do dos grupos populacionais ocidentais. Como resultado, o critério de IMC universal desenvolvidos pela Organização Mundial de Saúde (OMS) não é adequado para a população chinesa e outras populações asiáticas. ${ }^{17,18}$ Da mesma forma, o tamanho de efeito entre o diâmetro do átrio esquerdo e a mortalidade por IC entre a população chinesa e a população ocidental pode variar, mostrando que caucasianos geralmente têm um diâmetro do átrio esquerdo maior. ${ }^{19}$ Entretanto, estudos e explorações posteriores são necessários para quantificar essas diferenças. O presente escore foi desenvolvido usando um método estatístico robusto e foi validado utilizando-se dados adicionais. Também houve uma alta concordância com os resultados de derivação. Estas cinco presentes variáveis parecem atender aos critérios de variável ideal. Elas não são afetadas por interpretações clínicas, são amplamente aceitas, estão disponíveis no momento da internação, e podem ser facilmente coletadas. ${ }^{20}$ Por último, ao identificar os componentes modificáveis do presente escore de risco, os serviços de saúde pública podem ser desenvolvidos para resolver esses problemas específicos. Considerando a extraordinariamente grande população da China, esse escore poderia permitir o uso direcionado de recursos médicos e de saúde pública que não inevitavelmente limitados.

O presente estudo tem várias limitações comumente observadas no desenvolvimento de escores de risco baseados em dados clínicos. Isso não leva em conta fatores, tais como a qualidade do médico e do cuidado hospitalar, influências

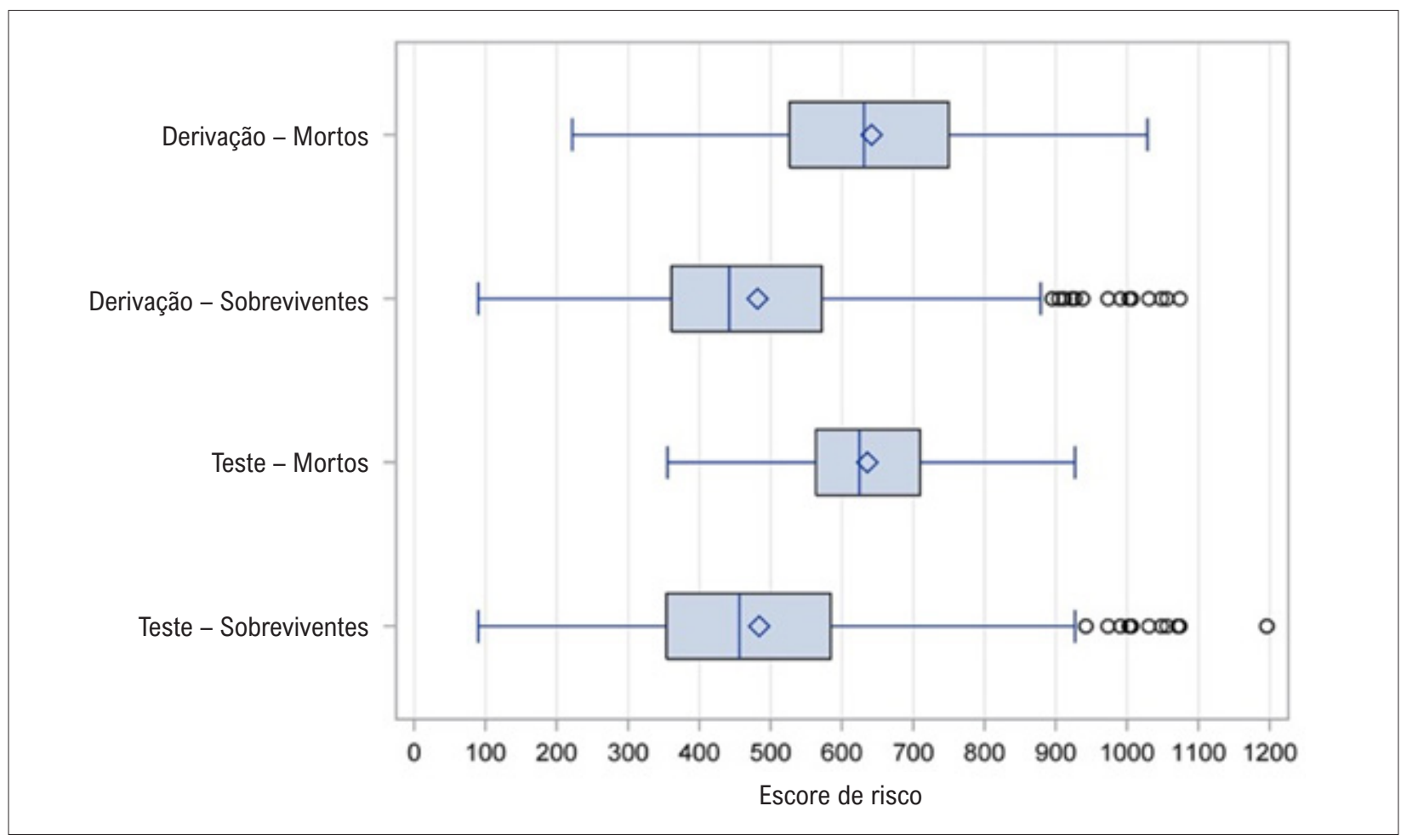

Figura 3 - Distribuição do escore de risco entre os grupos de óbito e sobreviventes. 
socioeconômicas, ou acesso ao cuidado. Os limiares de risco baixo, intermediário e alto foram baseados naquilo que os pesquisadores consideraram ser risco aceitável dentro de cada categoria. Além disso, informações importantes sobre tratamento, tais como tratamentos farmacológicos e por intervenção, incluindo inibidores de enzima de conversão de angiotensina, bloqueadores de receptores da angiotensina, betabloqueadores, e diuréticos, marca-passos biventriculares, e cardioversores desfibriladores implantáveis, não foram incluídos no modelo. Exames laboratoriais, tais como níveis de hemoglobina e sódio, não foram incluídos no modelo. Finalmente, os pacientes amostrados estavam em 14 hospitais em Pequim, uma das maiores cidades da China. Pode haver diferenças entre as características e o cuidado desses pacientes com IC em relação a residentes de áreas mais rurais ou remotas, que não são levados em consideração. Esse é um achado que foi observado ao se avaliar o risco cardiovascular na China. ${ }^{21}$ Além disso, 42\% dos pacientes têm classificação NYHA >3, e o índice de mortalidade é de apenas 6,5\%, o que deve ser avaliado em mais detalhes em detalhes futuros. No entanto, embora vários fatores clínicos não tenham sido incorporados à análise, o objetivo do estudo foi desenvolver uma ferramenta simples com variáveis facilmente definíveis para ajudar os médicos.

O presente escore pode ser utilizado para identificar pacientes de alto risco para garantir melhor cuidado após a alta. Dessa forma, ele pode ser usado como guia para médicos planejarem o cuidado de pacientes com IC, derivado de resultados baseados em evidências de pesquisas relevantes e confiáveis. Portanto, os resultados do presente estudo oferecem uma abordagem ao cuidado de saúde, que promove a coleta, a interpretação, e a integração de evidências válidas, importantes e aplicáveis, que estão relacionadas ao paciente, são observadas pelo clínico, e são derivadas de pesquisa, além do uso dessas evidências para ajudar a tomada de decisão.

\section{Conclusão e direcionamentos futuros}

Foi demonstrado um modelo para desenvolvimento de um escore de risco que é diretamente aplicável a pacientes chineses com IC. Isso poderia garantir melhor que fatores genéticos e ambientais de um grupo específico sejam levados em consideração e possam ser usados como estrutura para o desenvolvimento de escores de riscos em outros grupos raciais e étnicos.

\section{Contribuição dos autores}

Concepção e desenho da pesquisa e Redação do manuscrito: Wang L, Yun-Feng X; Obtenção de dados: Li-Qin W, Mo-Li G; Análise e interpretação dos dados: Li Liang; Análise estatística: Li-Qin W, Li Liang, Wang C; Obtenção de financiamento: Mo-Li G, Wang C; Revisão crítica do manuscrito quanto ao conteúdo intelectual importante: Wang L, Mo-Li G, Li Liang, Yun-Feng X.

\section{Potencial conflito de interesse}

Não há conflito com o presente artigo

\section{Fontes de financiamento}

O presente estudo não teve fontes de financiamento externas.

\section{Vinculação acadêmica}

Não há vinculação deste estudo a programas de pós-graduação.

\section{Aprovação ética e consentimento informado}

Este estudo foi aprovado pelo Comitê de Ética do Fourth Medical Center of PLA General Hospital sob o número de protocolo 2020KY002-KS001. Todos os procedimentos envolvidos nesse estudo estão de acordo com a Declaração de Helsinki de 1975, atualizada em 2013. O consentimento informado foi obtido de todos os participantes incluídos no estudo.

\section{Referências}

1. Jiang $\mathrm{H}, \mathrm{Ge}$. Epidemiology and clinical management of cardiomyopathies and heart failure in China. Heart. 2009;95(21):1727-31.

2. Kaul P, McAlister FA, Ezekowitz JA, Grover VK, Quan H. Ethnic differences in 1-year mortality among patients hospitalised with heart failure. Heart 2011;97:1048-53.

3. Vaughan-Sarrazin MS, Hannan EL, Gormley CJ, Rosenthal GE. Mortality in Medicare beneficiaries following coronary artery bypass graft surgery in states with and without certificate of need regulation. Jama 2002;288(15):1859-66.

4. Hannan EL, Stone CC, Biddle TL, DeBuono BA. Public release of cardiac surgery outcomes data in New York: what do New York state cardiologists think of it? Am Heart J. 1997;134(1):1120-8.

5. Murphy GJ, Ascione R, Caputo M, Angelini GD. Operative factors that contribute to post-operative atrial fibrillation: insights from a prospective randomized trial. Card Electrophysiol Rev. 2003;7(2):136-9.

6. Mathew JP, Fontes ML, Tudor IC, et al. A multicenter risk index for atrial fibrillation after cardiac surgery. JAMA. 2004;291(14):1720-9.
7. Davila-Roman VG, Kouchoukos NT, Schechtman KB, Barzilai B. Atherosclerosis of the ascending aorta is a predictor of renal dysfunction after cardiac operations. J Thorac Cardiovasc Surg. 1999;117(1):111-6.

8. Barzi F, Patel A, Gu D, Sretara P, Lam T, Asia Pacific Comunityet al. Cardiovascular risk prediction tools for populations in Asia. J Epidemiol Comm Health. 2007;61(2):115-21.

9. Wang Y. A Multinomial Logistic Regression Modeling Approach for Anomaly Intrusion Detection. Computers and Security 2005;24:662-74.

10. Hosmer D, Lemeshow, S. Applied logistic regression, 2nd ed Philadelphia: Wiley \& Sons, Inc.; 2000.

11. Palaniappan LP, Wong EC, Shin JJ, Fortmann SP, Lauderdale DS. Asian Americans have greater prevalence of metabolic syndrome despite lower body mass index. Int J Obes (Lond) 2011;35(3):393-400.

12. Palaniappan LP, Araneta MR, Assimes TL, Barrett- Connor El,Carnethon E, Criqui $\mathrm{M}$, et al. Call to action: cardiovascular disease in Asian Americans: a science advisory from the American Heart Association. Circulation 2010;122(12):1242-52. 
13. Adams KF, Jr., Sueta CA, Gheorghiade M, O'Connor CM, Schwartz TA, Koch AA. et al. Gender differences in survival in advanced heart failure. Insights from the FIRST study. Circulation 1999;99(14):1816-21.

14. Oreopoulos A, Padwal R, Kalantar-Zadeh K, Fonarow GC, Norris CM, McAlister FA. Body mass index and mortality in heart failure: a meta-analysis. Am Heart J.2008;156(1)13-22.

15. Rossi A, Temporelli PL, Quintana MF, Ghio S, Hillis G, Dini F, et al. Independent relationship of left atrial size and mortality in patients with heart failure: an individual patient meta-analysis of longitudinal data (MeRGE Heart Failure). Eur J Heart Fail .2009;11(10):929-36.

16. O'Connor CM, Whellan DJ, Wojdyla D, Leifer E, Clark RM, Fini RJ, et al. Factors related to morbidity and mortality in patients with chronic heart failure with systolic dysfunction: the HF-ACTION predictive risk score model. Circ Heart Fail.2012;5(1):63-71.

17. World Health Organization. (WHO). Obesity: preventing and managing the global epidemic: WHO technical report series n.o 894. Geneva;2000.
18. Shiwaku K, Anuurad E, Enkhmaa B, Kitajima K, Yamane Y, Appropriate BMI for Asian populations. Lancet -.2004; 363(9414):1077. DOI: 10.1016/S0140-6736(04)15856-X

19. Gottdiener JS, Reda DJ, Williams DW, Materson BJ. Left atrial size in hypertensive men: influence of obesity, race and age. J Am Coll Cardiol. $1997 ; 29(3): 651-8$

20. Radford MJ, Heidenreich PA, Bailey SR, et al. ACC/AHA 2007 Methodology for the Development of Clinical Data Standards: A Report of the American College of Cardiology/American Heart Association Task Force on Clinical Data Standards. J Am Coll Cardiol. 2007;49(7):830-7.

21. Gu D, Gupta A, Muntner P, Hu S, Suan X, Chen J, et al. Prevalence of cardiovascular disease risk factor clustering among the adult population of China: results from the International Collaborative Study of Cardiovascular Disease in Asia (InterAsia). Circulation 2005;112(5):658-65. 